\title{
Intelligent Eating to Support Mitochondrial Function
}

\section{Jennifer Gantzer*}

Department of Clinical Sciences, National University of Health Sciences, USA

*Corresponding Author: Jennifer Gantzer, Department of Clinical Sciences, National University of Health Sciences, USA.
Received: September 10, 2021

Published: October 01, 2021

(C) All rights are reserved by Jennifer Gantzer.
Intelligent Eating includes purposely choosing food for meals and snacks which support the body's metabolic demands maximizing its amazing intrinsic abilities to repair and regenerate. Intelligent Eating optimizes nutritional dietary intake which maximizes cellular processes at the molecular level supporting optimal function which then prevents and slows aging and degeneration. Instead of eating healthy which many people WANT to do but don't always know how to, I hope to shift the perception of eating healthy and the idea of dieting for health into the concept of Intelligent Eating as a Dietary Lifestyle where it's a normal part of everyday, not a fad or a diet, thereby to support repair and regeneration, sustain anti-aging, and restore and/or improve quality of life in ourselves, our families, and our patients.

Intelligent Eating sustains Mitochondrial Function; the cellular powerhouse the "mitochondria" to generate the "molecule of energy" known as ATP, Adenosine Triphosphate [1,2]. When the body and/or the cell stops being able to efficiently convert nutrition into energy as ATP, it's appropriately called "Mitochondrial Dysfunction" which has been clinically correlated to many disease states, especially the chronic, degenerative, and inflammatory ones and when it's reversed, disease states regress, and quality of life is restored $[3,4]$.

To sustain continual generation of cellular ATP we convert the macronutrients Carbs, Fats, and Proteins into it through a series of Essential Nutrient Vitamin and Mineral dependent enzymatic reactions. Essential Nutrients are dietary components required to fulfil physiological roles that must be ingested since we cannot make them on our own yet need them. Since we need ATP every moment of every day in every living cell we need to generate ATP from macronutrients whether we just ate them or we stored them away and will break them down to retrieve them in between meals. To enzymatically convert macronutrients into ATP we require all the essential B-vitamins plus the Essential Minerals Iron, Sulfur, Copper, Zinc, Magnesium, Molybdenum, Manganese and to support glucose entry into skeletal muscle also the Ultratrace Essential Minerals Chromium and Vanadium.

We additionally need all 20 physiological amino acids, the basic units of proteins, of which 9 are essential and several more of the 20 can become conditionally essential when their physiological demand increases since these are the building blocks we need to build the shuttles and the ATP machinery, which are proteins $[1,2,5]$. It's vital to nutritionally sustain all the Essential Nutrients every day with Intelligent Eating Dietary Lifestyle strategies.

How do we maximize the intake of Essential Nutrients and macronutrient conversion to ATP with dietary choices? Food sources the highest in essential Vitamins and Minerals are primarily from plant-based sources including fruits, vegetables, legumes, nuts, seeds, and whole grains $[1,2,6]$ and I strongly encourage a daily intake of phytogreen powders intermixed with alkalizing effervescent mineral and vitamin powders. While including and increasing our plant-based sources, we also must sustain protein intake since the amino acids we need to build the machinery and shuttle important nutrients and hormones around the body depend on them and furthermore the body's protein demand can double to triple during times of injury, infection, and inflammation [5]. Vegans and Vegetarians must be conscious of their plantbased protein sources and I recommend supplementing daily with a protein powder scoop. Alternatively, individuals on GERD medication or regular antacid ingestion also need to be aware some protein maldigestion occurs from these and there may be a 
protein deficit, and again I strongly encourage ingestion of a protein powder daily to ensure an optimal body balance of these vitally important building blocks $[1,5,6]$. While encouraging the ingestion of more plant-based dietary nutrient food choices for vitamins/ minerals and ensuring adequate protein it's best to avoid the foods that are debunk of these nutrients especially processed foods such as boxed or bagged items including many chips, crackers, cookies, many frozen or ready-made meals including microwaveable or tv dinners $[1,6]$. It's best to food prep as many of your own meals as possible, include as many different colored fruits and vegetables at each meal, and include spices and herbs which offer additional gut microbiome health benefits alongside antioxidants [7].

\section{Bibliography}

1. Gropper SS and Smith JL. "Advanced Nutrition and Human Metabolism". 6th ed. Wadsworth: Cengage Learning (2013).

2. Linus Pauling Institute, Oregon State Micronutrient Center.

3. Muravchick S and Levy RJ. "Clinical implications of mitochondrial dysfunction”. Anesthesiology 105.4 (2006): 819-837.

4. Natarajan V., et al. "Mitochondrial Dysfunction in Age-Related Metabolic Disorders". Proteomics 20.5-6 (2020).

5. Fürst P. "Basics in clinical nutrition: Proteins and amino acids". European e-Journal of Clinical Nutrition and Metabolism 4.2 (2009): e62-e65.

6. Richter Amy. "How do processed foods affect your health". MedicalNewsToday.com (2020).

7. Nakatani N. "Phenolic antioxidants from herbs and spices". Biofactors 13.1-4 (2000): 141-146.

Volume 4 Issue 11 November 2021

(C) All rights are reserved by Jennifer Gantzer. 